\title{
Image Processing in Contact Endurance Research
}

\author{
Andrey Kirichek ${ }^{1}$, Sergey Barinov ${ }^{2}$ and Aleksandr Yashin ${ }^{2}$ \\ ${ }^{1}$ Bryansk State Technical University, 50 let Octyabrya Blvd. 7, 241035, Bryansk, Russia \\ ${ }^{2}$ Vladimir State University, Orlovskaya Str. 23, 602264, Murom, Russia
}

\begin{abstract}
Machine parts operating under contact cyclic loads are subject to a destruction risk in the event of their working surface pitting (destruction). To increase the service life of such parts, various finishing and hardening technologies are used. In the absence of standard methods used to assess the durability of parts operating under contact endurance conditions, they have should be developed in relation to a specific case. The fractal geometry methods' usage in analyzing the surface layer state of parts operating under contact endurance conditions is complicated due to high equipment cost and fine and coarse noise high risk, which negatively affects the quality of generated images. The aim of the work is to develop a simple and accessible method for evaluating the results of contact endurance tests, based on the analysis of the investigated surface images. The developed patented technique consists of several stages. At the first stage, high-resolution photographing of the surface under investigation is performed using a microscope, providing magnification of no more than $1 \times 50$. At the second stage, the obtained image is analyzed in the cad editor, where the number and area of the investigated defects are determined. At the third stage, wear result numerical assessment is carried out according to the proposed algorithm.
\end{abstract}

\section{Keywords}

Image analysis, high image resolution, contact endurance, cad editor, pitting.

\section{Introduction}

The working surfaces of parts that perceive concentrated cyclic contact loads usually break down due to fatigue failure. Contact fatigue is the damage accumulation process and the development of the surface layers destruction of a material under alternating contact stresses action, which cause pitting or cracks formation as well as a decrease in durability. Contact fatigue typically causes the failure of gears, rolling bearings, rails and rail wheels, rolling rolls, cam pushers, strikers, worm gear, hypoid, screw, chain and globoidal gears, guides, spline joints with rolling elements, overrunning roller couplings etc.

To increase the durability of machine parts operating under contact fatigue loading, various hardening methods are usually used (heat treatment, chemical heat treatment, surface plastic deformation, etc.) aimed at increasing the surface layer hardness. Hardening effectiveness evaluation is possible only due to experimental tests. At present, R 50-64-30-87 recommendations are known but there is no standard describing a unified approach to equipment and methods for contact endurance assessment [1]. In this regard, researchers independently develop various methods and installations. Their research reliability primarily depends on how accurately test conditions reproduce the mating surface operating conditions.

To assess the durability of machine parts, which fail due to contact load action, various parameters are used.

GraphiCon 2021: 31st International Conference on Computer Graphics and Vision, September 27-30, 2021, Nizhny Novgorod, Russia EMAIL: avk.57@yandex.ru (A. Kirichek); box64@rambler.ru (S. Barinov); yashin2012@yandex.ru (A. Yashin) ORCID: 0000-0002-3823-0501 (A. Kirichek); 0000-0002-1341-446X (S. Barinov); 0000-0002-3186-1300 (A. Yashin)

(c) (i) (C) 2021 Copyright for this paper by its authors.

Use permitted under Creative Commons License Attribution 4.0 International (CC BY 4.0).

CEUR Workshop Proceedings (CEUR-WS.org) 
Contact fatigue is the ability of the contracting parts' working surfaces to withstand contact fatigue. The contact endurance quantitative assessment is the contact pressure value, which does not lead to pitting for a given number of cycles.

Often machine parts' durability, which fail due to the action of contact stresses, i.e. fatigue life, is assessed by the number of loading cycles during which pitting appears on the surfaces of parts.

Fatigue failure under cyclic contact loading occurs due to the accumulation of stresses in the surface layer under the action of normal forces periodically applied to the surface, which first promote cracks initiation and then their development. The emerging fatigue crack can join with adjacent microcracks. The path, as well its development speed, depend on the orientation of the microcracks encountered near it, as well as on mechanical properties of adjacent metal sections. Several fatigue cracks can develop in a part at the same time. After a certain number of loading cycles, the crack found under the conditions most conducive to its growth reaches the part surface. Then metal pitting occurs (with simultaneous crack propagation parallel to the part surface), as, for example, in Figure 1. Further part loading leads to the development of fatigue pitting [1].

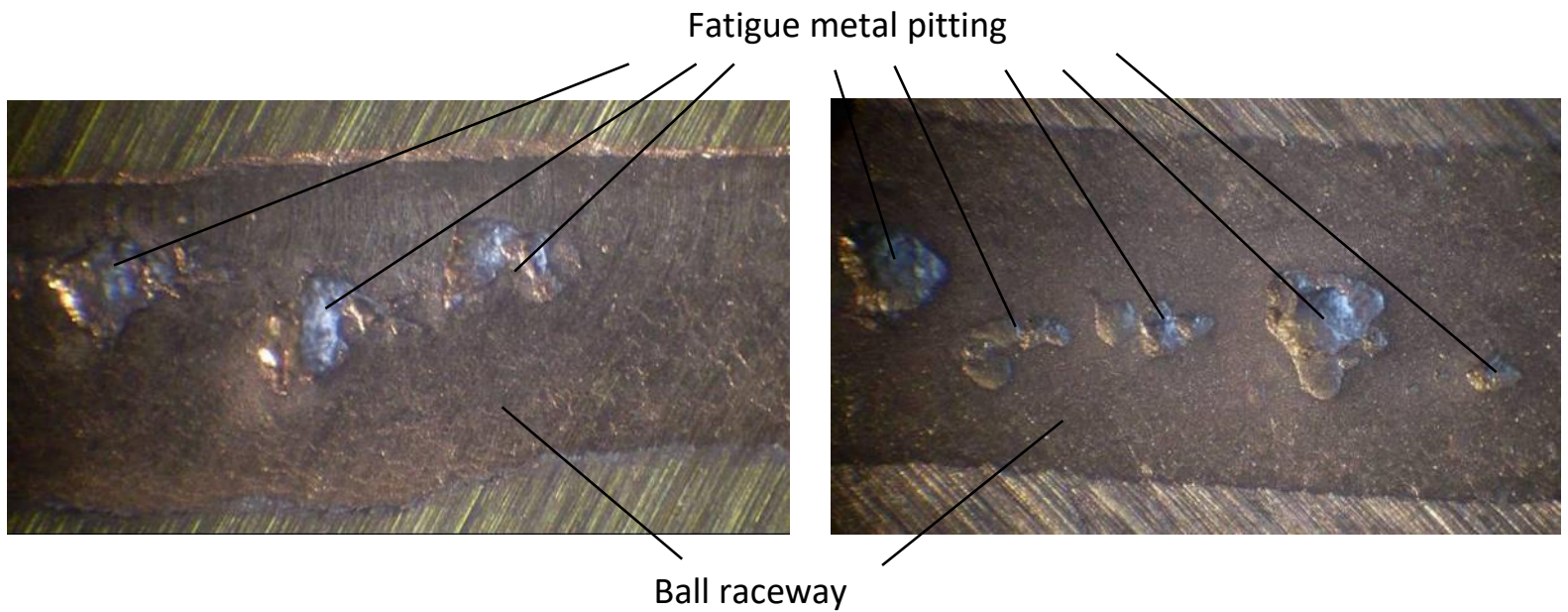

Figure 1: Examples of fatigue metal pitting on ball raceway at $1 \times 50$ magnification.

The criteria for contact fatigue fracture assessment are:

- reaching the diameter of one or several chipping pits of the calculated contact spot minor semiaxis half;

- contact area reduction to the limiting value according to the static strength criterion, i.e. before the beginning of plastic crushing of the spalling pits boundaries;

- the pits' area reaches a certain part of the tested surface total area;

- the chipping pits' appearance along any contact surface generatrix entire length or the achievement of the established pits' limit number at a certain generatrix length;

- reducing the sample mass until the specified value is reached.

Indirect criteria for contact fatigue tests are: changes in the noise pitch, vibration intensity, and the rotation resistance moment value.

Recently, the surface layer state parameters assessment is carried out on the analysis of images of the investigated (working) product surface by the fractal geometry methods [2-6]. With their help, research is carried out on the surface roughness of varying complexity geometric shapes and spatial microstructure [7,8]. However, the equipment used for non-contact research (as a rule, scanning tunneling microscopes), in addition to its high cost and requirements for high qualifications of the operator, has a number of other disadvantages. The disadvantages are connected with the complexity of equipment isolation from mechanical and acoustic high-frequency vibrations, leading to fine and coarse noise appearance in the generated images.

The purpose of this work is to develop an accessible technique for studying contact endurance based on the investigated surface image processing. 


\section{Image processing stages in the contact endurance study}

The main principles underlying the contact endurance assessment methodology based on image analysis are: implementation ease, assessment accuracy and instrumental availability [9].

The investigated surface with traces of wear in the form of pitting is divided into sectors. The size of a single sector is determined by the used microscope optical capabilities. For this kind of research, equipment with $1 \times 50$ magnification is sufficient. The equipment with such optical characteristics is widely used in laboratories (for example, instrumental microscopes).

Through the microscope objective, high-resolution photographs of the investigated surface selected sectors having wear traces are taken.

The resulting images are transferred to a specialized software package, for example, a CAD system (Compass, AvtoCAD or SolidWorks). In the CAD system, using digital photographs of sectors and their linear dimensions' values, images of sectors are processed at 1: 1 scale. On the images obtained, the sectors' boundaries and the existing contact-fatigue surface pitting are outlined. They are shown in Figure 2. The outlined contours are used to determine their size, number and area. An example of pitting contours' area estimation in the CAD system is shown in Figure 3.

Based on the image analysis of each test sample, the following are determined: the pitting area $S$ $\left(\mathrm{mm}^{2}\right)$; raceway area $S_{\text {race }}\left(\mathrm{mm}^{2}\right)$, number of spalls $n$ (pcs.), maximum $S_{\max }\left(\mathrm{mm}^{2}\right)$ and minimum $S_{\min }$ $\left(\mathrm{mm}^{2}\right)$ areas of spalls.

For a qualitative and quantitative hardening effectiveness assessment, indicators that make it possible to compare the size and number of spalls in the hardened and non-hardened areas have been introduced:

Relative number of pitting

$$
\Delta n=\frac{S_{d}}{S_{d 0}} \cdot \frac{n_{0}}{n},
$$

where $n$ - the number of pitting in the investigated area, pcs; $S_{\mathrm{d}}$ - the balls' raceway area in the investigated sector, $\mathrm{mm}^{2}$ (" 0 " index means that the parameters characterize the unhardened surface).

The relative area of pitting

$$
\Delta S=\frac{\sum S_{0}}{\sum S} \cdot \frac{n_{0}}{n},
$$

where $\Sigma S$-total pitting area in the investigated sector, $\mathrm{mm}^{2}$.

Minimum relative pitting area

$$
\Delta S_{\text {min }}=\frac{S_{0 \text { min }}}{S_{\text {min }}},
$$

where $S_{\min }$ - least pitting area measured in the test area, $\mathrm{mm}^{2}$.

Maximum relative pitting area

$$
\Delta S_{\max }=\frac{S_{0 \max }}{S_{\max }},
$$

where $S_{\max }$ - maximum pitting area, measured in the investigated sector, $\mathrm{mm}^{2}$.

Resistance to contact pitting $\Delta I$ is taken as the main characteristic that allows us to assess the hardened specimens' durability, which makes it possible to qualitatively and quantitatively compare the pitting amount on a hardened surface relative to an unhardened one.

$$
\Delta I=\frac{S_{d}}{S_{d 0}} \cdot \frac{\sum S_{0}}{\sum S} .
$$

Resistance to contact pitting, $\Delta I$, is a complex parameter that takes into account the change in the size of the tracks of the balls raceway during testing, and the pitting area that occurs on them, which makes it possible to objectively assess the durability of machine parts operating under contact fatigue conditions. 


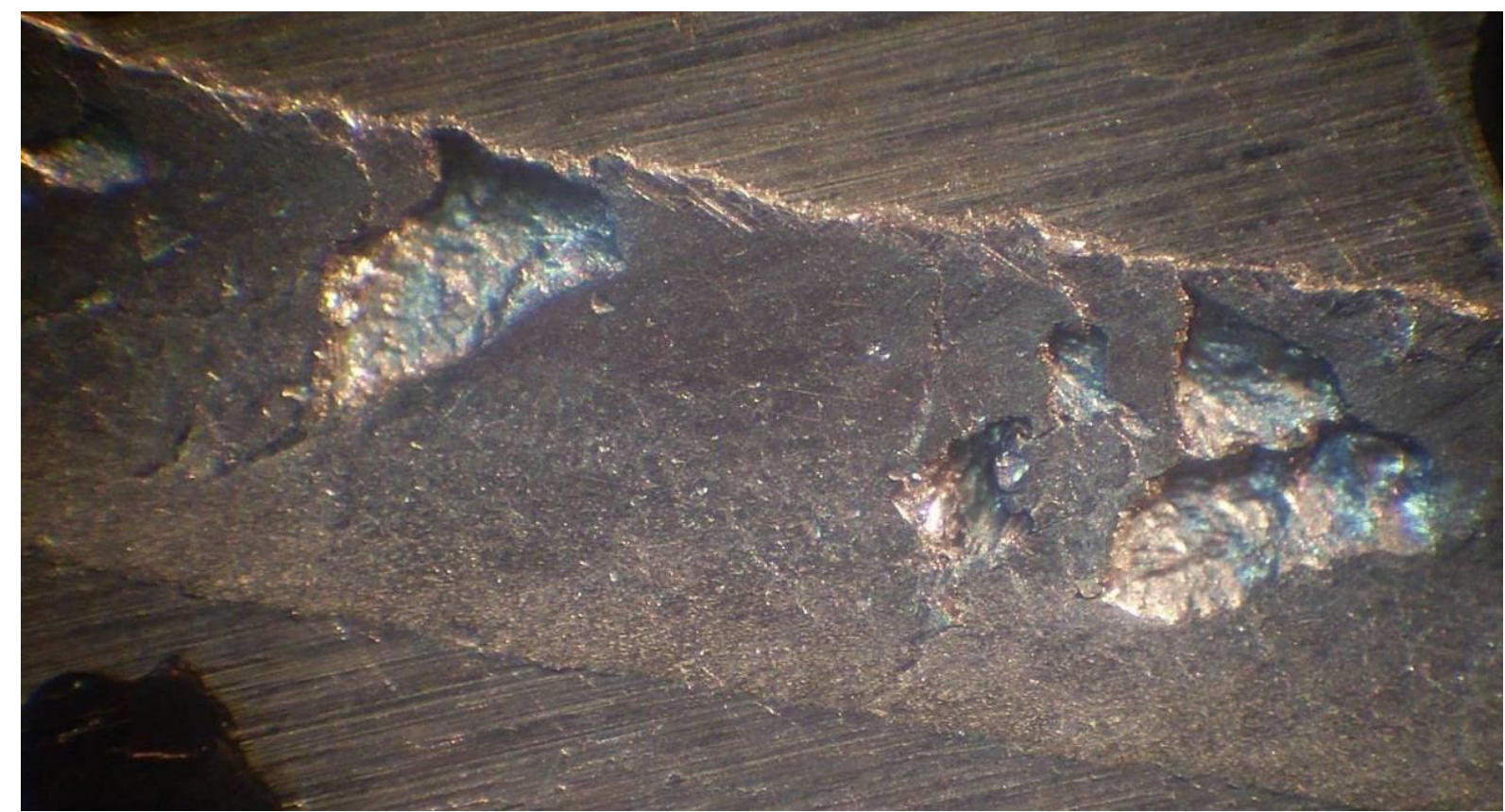

Figure 2: Maximum pitting area measured in the investigated sector, $\mathrm{mm}^{2}$ at $1 \times 50$ magnification

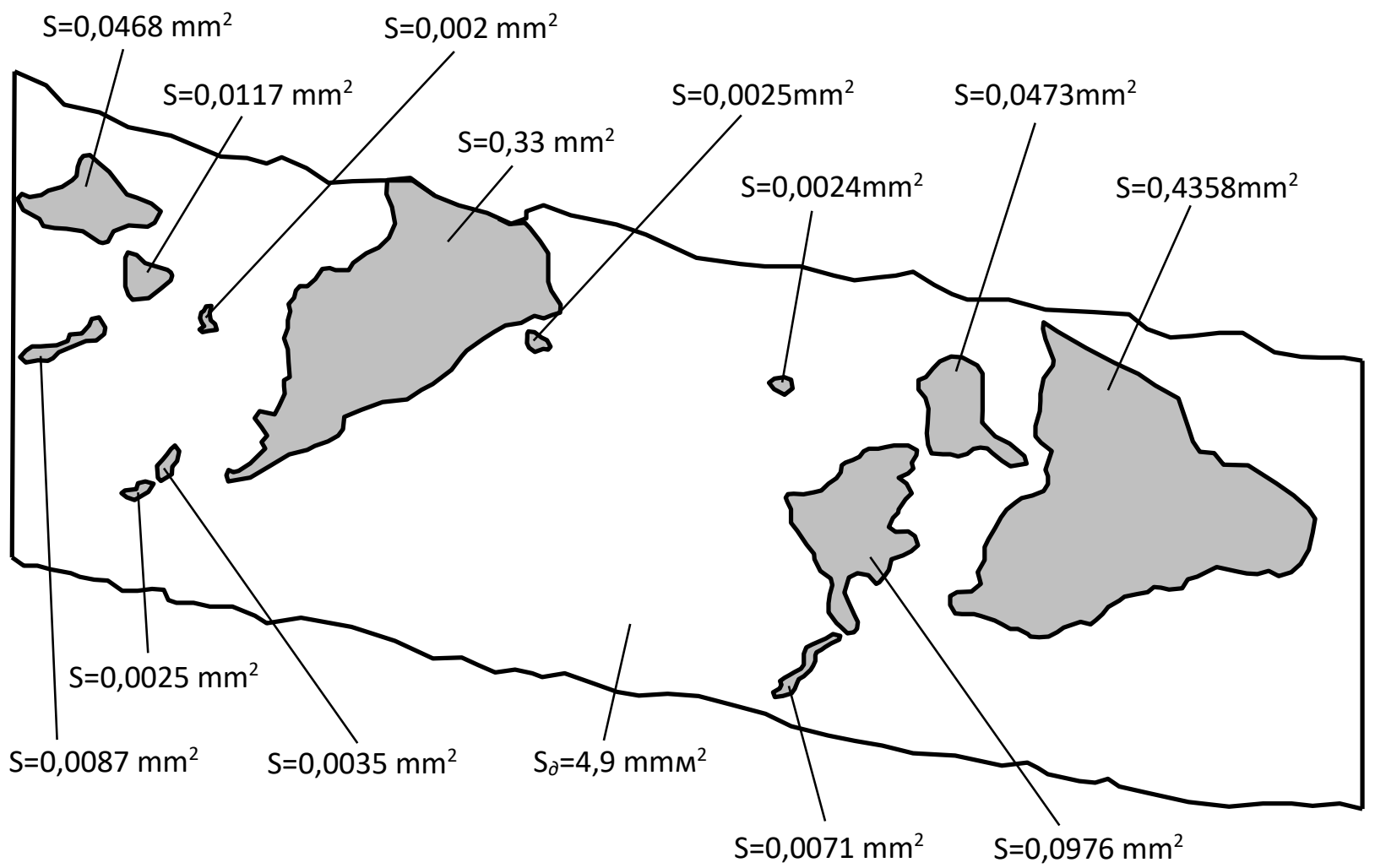

Figure 3: Pitting contours for estimating their area in CAD system.

\section{Conclusion}

The application of this technique made it possible to identify wave strain hardening rational modes, which allow us to create a heterogeneously hardened structure in the part surface layer, providing an increase in $\Delta I$ by 3-6 times, a decrease in the pitting number by 1.5-2.5 times and a decrease in pitting size by 2-3 times [10]. 


\section{References}

[1] A.V. Kirichek, Investigation of contact pitting of machine parts' surfaces, Control and diagnostics in ensuring the quality of machine-building products, Spectr, Moscow, 2012.

[2] G.A. Edgar, Measure, topology and fractal geometry, Springer-Verlag, New York, NY, 1990.

[3] J.C. Russ, Fractal Surface, Plenum Press, New York and London, 1994.

[4] M. Navascués, A. Chand, V. Veedu, and M. Sebastián, Fractal Interpolation Functions: A Short Survey, Applied Mathematics 5 (2014) 1834-1841. doi: 10.4236/am.2014.512176.

[5] J. Gleick, Chaos: Making a new science, Viking penguin, 1987.

[6] R. Slobodrian, Mathematical and Physical Fracta, Applied Mathematics 5 (2014) 1791-1800. doi: 10.4236/am.2014.512172.

[7] K. Arakawa, E. Krotkov, Fractal surface reconstruction uncertainty estimation: modeling natural terrain, School of Computer Science Carnegie Mellon University, 1992.

[8] M. Arafah, Q. Moghli, Efficient Image Recognition Technique Using Invariant Moments and Principle Component Analysis, Journal of Data Analysis and Information Processing 5 (2017) 110. doi: 10.4236/jdaip.2017.51001.

[9] Yu.S. Stepanov, A.V. Kirichek, D.L. Soloviev, S.A. Silantyev, S.V. Barinov, B.I. Afanasyev, D.E. Tarasov, D.S. Fomin, Method for contact endurance testing, 2009, Patent No. 2357228, Filed July 1st., 2008, Issued Aug. 9th., 2009.

[10] A.V. Kirichek, S.V. Barinov, Study of methods relating to increase of contact pitting resistance in $45,40 \mathrm{H}, 35 \mathrm{HGSA}$ steel due to development of heterogeneous structure involving mechanical hardening technique, Applied Mechanics and Materials 756 (2015) 65-69. doi: 10.4028/www.scientific.net/AMM.756.65. 